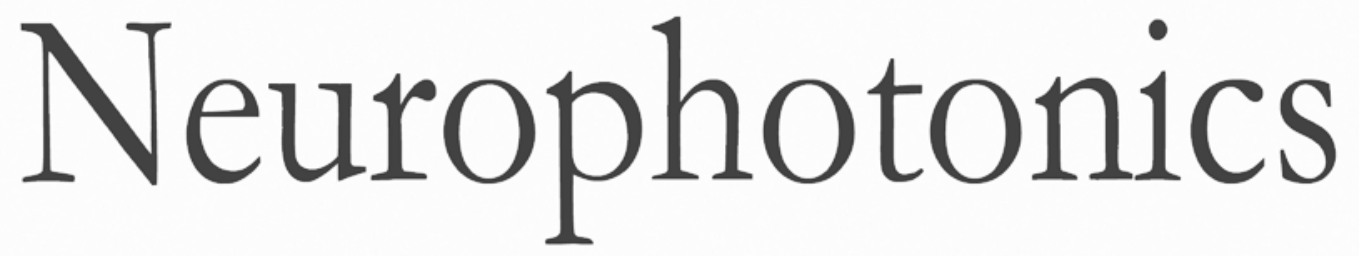

\title{
Review of combined isotopic and optical nanoscopy
}

Katharina N. Richter

Silvio O. Rizzoli

Sebastian Jähne

Angela Vogts

Jelena Lovric 


\title{
Review of combined isotopic and optical nanoscopy
}

\author{
Katharina N. Richter, ${ }^{a, *}$ Silvio O. Rizzoli, ${ }^{a}$ Sebastian Jähne, ${ }^{a, b}$ Angela Vogts, ${ }^{c}$ and Jelena Lovric ${ }^{d}$ \\ aUniversity of Göttingen Medical Center, Cluster of Excellence Nanoscale Microscopy and Molecular Physiology of the Brain, \\ Center for Biostructural Imaging of Neurodegeneration, Department of Neuro- and Sensory Physiology, Göttingen, Germany \\ bInternational Max Planck Research School for Neurosciences, Göttingen, Germany \\ 'Leibniz-Institute for Baltic Sea Research, Rostock, Germany \\ ${ }^{\mathrm{d}}$ Chalmers University of Technology, Department of Chemistry and Chemical Engineering, Gothenburg, Sweden
}

\begin{abstract}
Investigating the detailed substructure of the cell is beyond the ability of conventional optical microscopy. Electron microscopy, therefore, has been the only option for such studies for several decades. The recent implementation of several super-resolution optical microscopy techniques has rendered the investigation of cellular substructure easier and more efficient. Nevertheless, optical microscopy only provides an image of the present structure of the cell, without any information on its long-temporal changes. These can be investigated by combining super-resolution optics with a nonoptical imaging technique, nanoscale secondary ion mass spectrometry, which investigates the isotopic composition of the samples. The resulting technique, combined isotopic and optical nanoscopy, enables the investigation of both the structure and the "history" of the cellular elements. The age and the turnover of cellular organelles can be read by isotopic imaging, while the structure can be analyzed by optical (fluorescence) approaches. We present these technologies, and we discuss their implementation for the study of biological samples. We conclude that, albeit complex, this type of technology is reliable enough for mass application to cell biology. ๑ The Authors. Published by SPIE under a Creative Commons Attribution 3.0 Unported License. Distribution or reproduction of this work in whole or in part requires full attribution of the original publication, including its DOI. [DOI: 10.1117/1. NPh.4.2.020901]
\end{abstract}

Keywords: super-resolution microscopy; secondary ion mass spectrometry; protein turnover; stimulated emission depletion microscopy; stochastic optical reconstruction microscopy; photoactivated light microscopy; NanoSIMS; correlated imaging.

Paper 16058SSVRR received Aug. 19, 2016; accepted for publication Apr. 10, 2017; published online Apr. $22,2017$.

\section{Introduction}

Imaging of cellular activity is of great importance for understanding how cells behave in a time-dependent manner and how they react to certain stimuli, however, attempts at visualizing cellular activity have not been satisfyingly successful so far. Electrophysiological recordings of neurons provide a good measurement of ion currents and thus the electrochemical activity, but do not create a spatial image of the cell's activity. In contrast, imaging approaches, such as various fluorescence microscopy techniques, are able to visualize cellular targets and their fate over a short time span using live imaging. Nevertheless, monitoring the turnover of such targets in the cell, thus visualizing cell activity, also requires long-term approaches. Imaging over time periods of weeks or even months is not feasible using fluorescence microscopy of living cells due to the induction of photodamage and eventually cell death. Other techniques, such as pulse-chase experiments, using radioactively labeled marker molecules, ${ }^{1}$ provide the opportunity for longterm monitoring, but most pulse-chase imaging tools exhibit a low spatial resolution. In contrast, electron microscopy gives excellent resolution, but the identification of individual proteins or organelles is difficult as protein labeling (e.g., with goldlabeled antibodies) is still inefficient.

To accurately measure turnover within the cell though, specific proteins need to be visualized while the fate of subcellular compartments needs to be assessed. Over the last few years, imaging tools have been developed for this purpose. To achieve a

*Address all correspondence to: Katharina N. Richter, E-mail: k.richter1@ stud uni-goettingen.de resolution that is suitable to localize proteins in the cell, imaging techniques had to be developed that were able to break the diffraction limit. Conventional microscopes, such as confocal laser scanning microscopes, are limited in their resolution to about $200 \mathrm{~nm}$ due to the diffraction of light, which occurs when a light wave interacts with an object. This physical phenomenon causes the image of a point-like object to be depicted in a concentric pattern (Airy disk pattern), which is much broader than the original object. Thus, if the Airy disk patterns of two objects overlap, they cannot be discriminated in the image generated by a conventional microscope. In the past 10 to 20 years, this diffraction resolution barrier has been broken by several super-resolution imaging techniques using different concepts of generating subdiffraction-limit resolution. In this review, we explain the principles behind several super-resolution imaging techniques. In addition, we describe "temporal imaging" tools, which can be used to investigate the past history of cells and organelles, by imaging their isotopic composition. Finally, we explain how they can be combined to provide a technique that is suitable to monitor mid- to long-term changes in cell activity, while identifying specific compartments and proteins in the cell.

\section{Super-Resolution Imaging Approaches}

\subsection{Concepts of Super-Resolution Microscopy}

Over the past 20 years, tremendous progress has been made in the development of imaging techniques that provide diffraction unlimited resolution. A number of such super-resolution imaging techniques have been developed that make use of the ability 
to switch fluorophores on and off (photoswitching/photoconversion). Although based on the same principle of photoswitching/ photoconversion, the different techniques rely on different implementations to attain subdiffraction resolution. These concepts can be divided roughly into two categories according to the main principle used to exploit photoswitching of fluorophores to achieve super-resolution: one employing the modulation of the point spread function (PSF), whereas the other uses a stochastic approach. The most prominent example for the first approach is stimulated emission depletion (STED) microscopy, which was awarded with the Nobel Prize in 2014. The diffraction pattern of an illuminated target, obtained by a microscope, exhibits a specific intensity distribution, the so-called PSF. The full width at half maximum (FWHM) of this PSF [see Fig. 1(a) for illustration] describes the microscope's resolution. Therefore, minimizing the PSF of a microscope by depleting a subset of excited fluorophores using stimulated emission describes one way of obtaining subdiffraction resolution.

The second principle used for super-resolution imaging is based on the ability to only activate a subset of fluorophores stochastically. Sequentially collecting the emission of only a sparse set of activated fluorophores makes it possible to localize those fluorophores with high precision. This approach is employed in super-resolution imaging techniques, such as stochastic optical reconstruction microscopy (STORM) and photoactivated light microscopy (PALM).

\subsection{Stimulated Emission Depletion Microscopy}

The principle of STED microscopy was published in 1994 by Hell and Wichmann. ${ }^{4}$ They were the first to describe the principle of STED as a tool to break the diffraction barrier. As mentioned earlier, STED microscopy is based on the modulation of the microscope's PSF relying on the principle of reversible saturable optical transition. ${ }^{5}$ This means the switching of a fluorophore between two different states (here, the excited and deexcited states) can be optically induced. In STED microscopy, the switching from an excited state to the nonfluorescent deexcited state is induced by stimulated emission. In general, an electron in the ground energy state $S_{0}$ can be excited by the absorption of a photon to the first excited electronic state $S_{1}$. The electron in the higher energy state can relax back into the ground state by emitting a photon, thus emitting fluorescence. This spontaneous emission of photons can be "depleted" by stimulated emission. Upon encountering a photon of a specific energy level, the electron is forced back into the ground energy state by emitting a photon of the same wavelength as the photon encountering the electron in the first place. Due to this forced emission of a photon, the electron cannot undergo spontaneous emission of a photon. As the photon forced to be emitted is of the same wavelength as the photon used for the STED effect, the wavelength can be chosen in such a way that it can be filtered out from the spontaneous fluorescence one wants to detect. In an STED microscope, this principle is realized by using two lasers

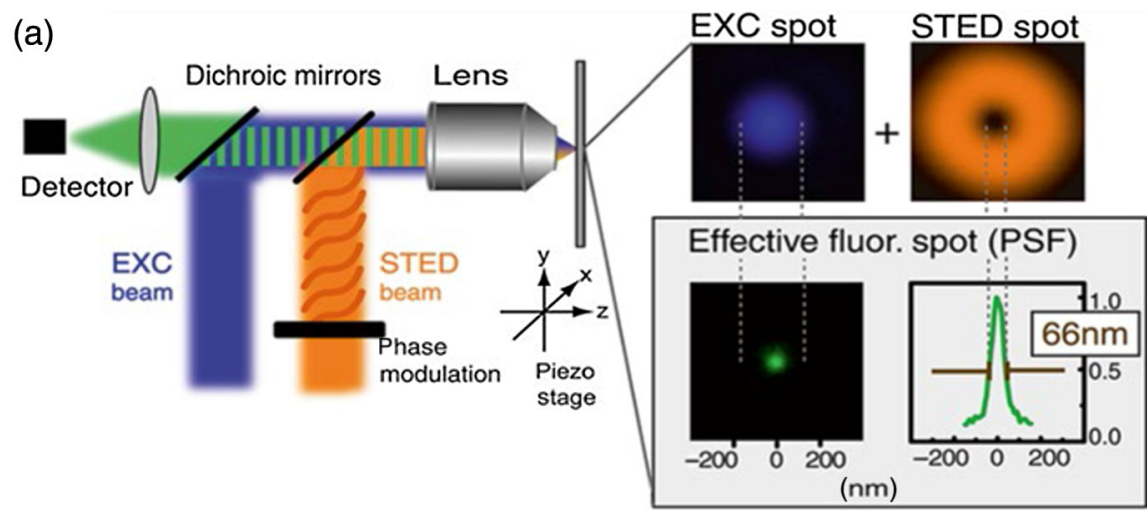

(b)

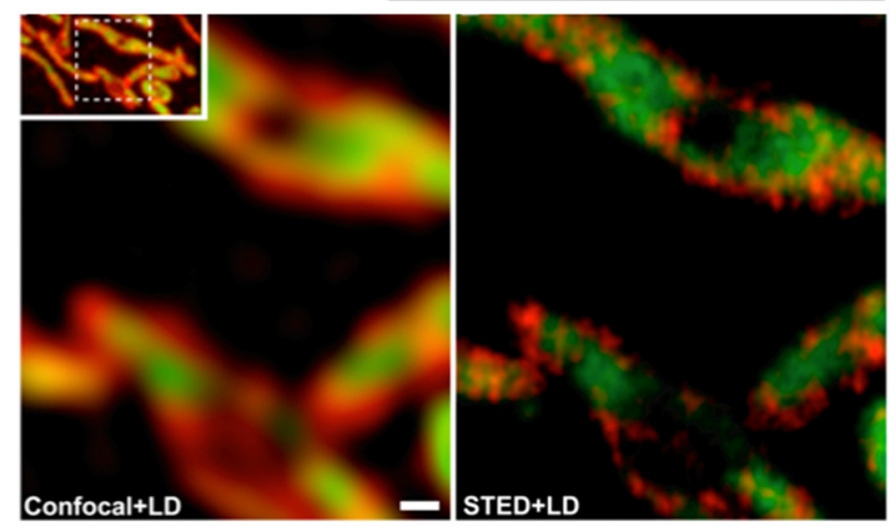

Fig. 1 (a) STED microscopy, principle of the technique. The excitation beam (blue) and the doughnutshaped STED beam (orange) overlap so the excited fluorophores in the periphery of the excitation beam are depleted. Thus, only fluorophores in the center, where the STED beam power is zero, are allowed to fluoresce. This leads to a fluorescent spot with an FWHM of $66 \mathrm{~nm}$ (green). ${ }^{2}$ (b) Comparison of confocal (left) and STED resolution (right). The mitochondrial protein Tom20 (red) and the F1F0 ATP synthase (green) were imaged, using antibody labeling. Two-color STED microscopy is able to resolve single Tom20 clusters, whereas conventional confocal microscopy is not. ${ }^{3}$ Scale bar: $200 \mathrm{~nm}$. Figures were reprinted with permission from Refs. 2 and 3. (c) 2007, Elsevier. 
that are aligned so they overlap. One of those is the excitation laser, used to excite the respective fluorophores as in a normal confocal microscope. The second laser beam is the STED beam, which is phase modulated to a doughnut shape and set to a longer wavelength than the excitation laser to deplete the fluorophores in the periphery of the excitation beam. This is done by stimulating the fluorophores to emit photons at this longer wavelength outside of the detection range of the microscope setup., This reduces the spot where fluorophores are allowed to fluoresce to the center of the overlapping excitation and STED beams, where the power of the STED laser is zero [Fig. 1(a)]. By increasing the power of the STED laser, the concentric area of depletion is increased, thus the fluorescent spot in the center is decreased. Thereby, a resolution can be achieved that is much higher than the resolution limit of conventional microscopes. The average resolution of an STED microscope, measured by the FWHM, by now is $\sim 60$ to $70 \mathrm{~nm}$, although a resolution of $<20 \mathrm{~nm}$ has been demonstrated as well. ${ }^{7,8}$ With a two-color STED setup, it is possible to image samples labeled with two different fluorophores, acquiring images in two separate channels with STED resolution. ${ }^{3,9}$ Using this setup, the authors of a colocalization study were able to resolve nanoclusters of the mitochondrial membrane protein Tom 20 in comparison to the $F_{1} F_{0}$ ATP synthase [Fig. 1(b)]. ${ }^{3}$ Another important tool in super-resolution imaging is provided by live-cell STED imaging. This was used for example by Nägerl et al. ${ }^{10}$ to monitor changes in the morphology of dendritic spines. Various studies have also shown that livecell STED imaging can be combined with a two-color setup. ${ }^{9,11}$ Live-cell imaging has been improved further by achieving videorate temporal resolution, which allowed to record synaptic vesicle movements in hippocampal neurons. ${ }^{12}$ Another STED-based application details the investigation of diffusion and interaction dynamics of fluorescently labeled molecules by combining STED with fluorescence correlation spectroscopy. ${ }^{13}$ All of the examples above clearly show that STED microscopy is an important tool to investigate versatile questions with a technique that allows resolving single protein clusters.

\subsection{Stochastic Optical Reconstruction Microscopy and Photoactivated Light Microscopy}

STORM and PALM also rely on the switching or conversion of fluorophores from the on-state to the off-state and vice versa. Their technical implementations are based on the stochastic activation of a small, randomly positioned subset of fluorophores at a given time. Iterative imaging of that subset and activation of a new subset of excited fluorophores lead to the accumulation of a series of images all containing the positions of the different fluorophores [the principle is shown in Fig. 2(a)]. The centers of those sparse, diffraction-limited spots in one image can be calculated by fitting to a Gaussian function. Thus, the position of each fluorophore can be determined with high precision, due to the lack of nearby interfering fluorophores. From the collected data, an overall image can be reconstructed. With this imaging technique, the resolution is mainly dependent on the density of the labeling and the number of photons one is able to collect from one fluorophore. Thus, with a high quantum yield and a low background noise, the localization precision can be very high, therefore, the resolution is not limited. Usually, a resolution (localization precision) of $\sim 20 \mathrm{~nm}$ is achieved with these imaging techniques.

Both techniques, STORM ${ }^{14}$ and PALM,${ }^{16}$ were described first in 2006 by separate research groups, although they both use the same main principle. Initially, STORM imaging was developed for antibody-based labeling with inorganic dyes, whereas PALM imaging was developed for labeling with fluorescent proteins.

One example of STORM-based investigations demonstrates the possibility to visualize the organization of pre- and postsynaptic proteins in mouse brain tissue with a localization precision of $14 \mathrm{~nm} .{ }^{17}$ Furthermore, an improvement in localization precision along the $z$-axis (with regard to conventional and even STED imaging) has been shown by the application of threedimensional (3-D)-STORM, achieving an axial resolution of 25 to $30 \mathrm{~nm} .{ }^{18,15}$ Combining 3-D STORM with a dual-color system by employing a second photoswitchable dye and a second laser, Jones et al. ${ }^{15}$ were able to localize transfer in the middle of clathrin coated pits. The comparison to conventional microscopy shows the immense improvement in the precision of colocalization along all three axes [Fig. 2(b)].

Two years after the emergence of STORM and PALM, in 2008, photoactivated localization microscopy was demonstrated to be suitable for live-cell imaging with an initially slow temporal resolution of $25 \mathrm{~s}$ per frame. ${ }^{19}$ Later, the use of STORM and PALM live-cell imaging with video-rate temporal resolutions as well has also been achieved. ${ }^{20}$

Taken together, all of the examples described above show that STORM and PALM present versatile tools to investigate cellular processes with a resolution in the nanometer range, even exceeding the high resolution of STED microscopy.

\subsection{Limitations of Super-Resolution Microscopy in the Temporal Domain}

STORM, PALM, and STED microscopy prove how immensely imaging techniques have been improved in the past years by breaking the diffraction barrier and thus providing subdiffraction resolution. The improvement in resolution and the possibility of multicolor and live imaging render those techniques suitable for a wide range of applications, although each exhibits a different set of advantages and limitations, making them suitable for distinct applications. One advantage of STED microscopy is the possibility to image relatively thick samples, as it is built on the principle of confocal microscopy. This is not the case for STORM/PALM microscopy, where imaging is limited to relatively thin samples unless it is combined with light sheet microscopy. While STORM/PALM imaging provides information on every molecule and thus allows for further analysis, such as counting of molecules, this amount of detailed information on each molecule is not provided in STED imaging. Nevertheless, by applying further data-processing methods, one can extract information about the number and distribution of molecules from STED images, as recently performed by Ta et al. ${ }^{21}$ One major limitation of STORM/PALM microscopy is the need for deconvolution of the raw data acquired by those imaging techniques, which limits the speed of STORM/PALM in contrast to STED imaging. STORM/PALM also face the limitation of background and noise signals, and the images have to be subjected to the careful correction of drift and chromatic aberration, and finally, the overall image has to be constructed. This amount of data processing not only makes the image production a slow process but is also a potential source of artifacts.

In addition to individual limitations of each technique, STED, STORM, and PALM further exhibit limitations that are shared by each of the described imaging techniques. The choice of dyes is limited by the need for extremely photostable and 
(a)

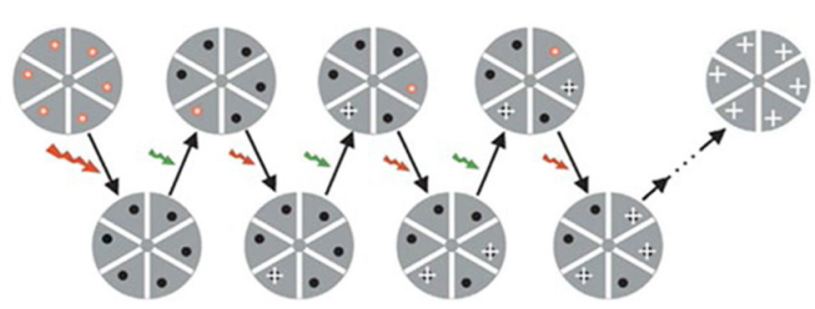

(b)
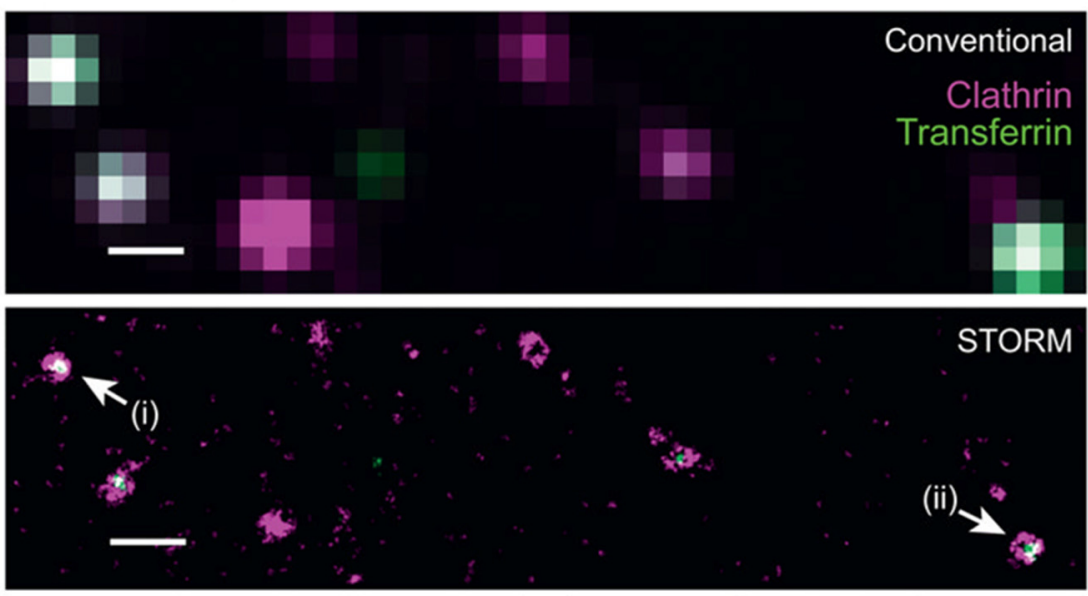

(c)
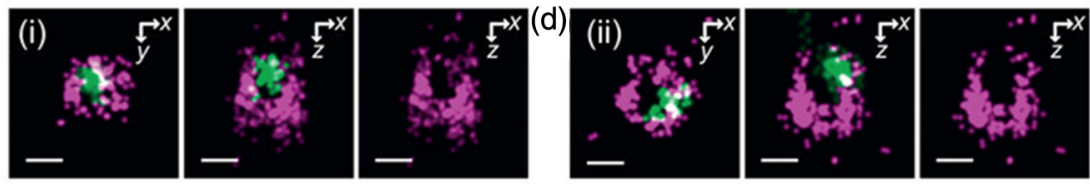

Fig. 2 (a) STORM and PALM, principle of STORM and PALM imaging. Present fluorophores in a sample are either in a dark state from the beginning or are turned off by a pulse (red arrow). Iterative activation of a subset of fluorophores and imaging of that subset lead to the accumulation of images, from which the precise locations of the molecules can be calculated. An overall image of all localized molecules can be constructed from this series of single images. ${ }^{14}(\mathrm{~b})$ Comparison of conventional and 3-D STORM images of clathrin coated pits (magenta) and transferrin (green). (c) and (d) Zoomed-in clathrin coated pits indicated in (b), showing the $x y$ projections (left panels) or $x z$ projections with (middle panels) and without (right panels) the transferrin label. ${ }^{15}$ Scale bars: (b) $500 \mathrm{~nm}$ and (c) and (d) $100 \mathrm{~nm}$. Scheme in (a) was reprinted with permission from Ref. 14. (C) 2006, Macmillan Publishers Ltd. Figures (b) to (d) were reprinted with permission from Ref. 15. @ 2011, Macmillan Publishers Ltd.

photoswitchable/photoactivatable fluorophores. This again leads to a limited spectrum of fluorophores that can be used in a multicolor experiment. Furthermore, live-cell imaging, especially for most STORM and PALM setups, still exhibits a poor temporal resolution regarding the investigation of fast cellular processes. Frame rates of about 20 to $30 \mathrm{~s}$ are not suitable to resolve processes such as synaptic vesicle release. Faster frame rates have been accomplished, but often at the expense of maintaining physiological conditions during long-term imaging. The utilization of faster cameras and appropriate localization algorithms is currently greatly improving temporal resolution, but possible long-term effects in terms of photo-toxicity on the samples might occur. Furthermore, super-resolution microscopy techniques are not able to monitor long-term changes in the cell, which is especially important for the investigation of cell activity, i.e., protein turnover. Here, photobleaching is one of the biggest challenges that limits long-term imaging to a few tens of minutes at most. ${ }^{19}$ Both STED and STORM/PALM operate with relatively high laser intensities, not only inducing photodamage but also increasing the irreversible bleaching of fluorophores in fixed cells as well as in live cells. In STED microscopy, the high intensity of the STED laser leads to rapid bleaching even of highly photostable dyes, such as Atto647. In STORM and PALM, slightly lower laser intensities are used, but those are still enough to bleach fluorescent proteins or dyes after continued imaging. Thus, the pool of available fluorescent molecules that can be activated will decrease, which in turn leads to a reduced signal-to-noise ratio. As the resolution in all these techniques depends on the number of photons that the fluorophore emits before bleaching, photobleaching will result in a drastic decrease in resolution. Nevertheless, many examples from recent years show that super-resolution microscopy techniques are important tools in the investigation of biological questions, closing the gap between conventional light microscopy and electron microscopy.

\section{Long-Term Changes: Pulse-Chase Labeling}

Super-resolution microscopy alone is not able to provide the answer to questions outside of localization and colocalization studies. A striking example of this is the identification of longterm protein turnover rates as an estimate for cell activity. As mentioned in Sec. 1, for monitoring cell activity, cellular compartments need to be identified, which can be done by super-resolution microscopy with very high precision, but for measuring protein turnover itself, other techniques are more suitable. Therefore, during the last few years, techniques have been established that combine imaging tools with protein identification for monitoring long-term changes. 
Many studies comprising protein identification and the monitoring of long-term changes employ pulse-chase labeling of the target molecule suitable for in-situ measurements. Pulse-chase experiments have shown a great importance, for example, in autoradiographic imaging studies, ${ }^{22}$ where target proteins are labeled during a "pulse" phase with a radioactive isotope usually incorporated in an amino acid. Contrary to autoradiography, the matrix-assisted laser desorption/ionization (MALDI) technique developed by Karas et al. ${ }^{23}$ in 1985 is a label-free mass spectrometry imaging technique, which allows mapping molecular ions with a high molecular weight. Application of a high-energy pulsed laser beam combined with an appropriate light adsorbing matrix makes MALDI a soft ionization technique with the ability to image, in parallel, large intact biomolecules, such as peptides and proteins. ${ }^{24,25}$ However, even though both techniques provide an opportunity to localize proteins in biological samples, their application in the field of subcellular imaging is not possible due to the low spatial resolution, ${ }^{22,24}$ which does not satisfy the need for a submicrometer range.

\subsection{Secondary Ion Mass Spectrometry Imaging}

A complementary technique, both to autoradiography and MALDI, is secondary ion mass spectrometry (SIMS), an imaging technique for acquiring atomic and molecular ion maps. ${ }^{26}$ SIMS with high mass and lateral resolution capabilities and parts per million or parts per billion detection limit is widely applied in biology. In SIMS analysis (Fig. 3), a focused primary ion beam is used to sputter material from a sample and produce secondary ion species, which are analyzed with a mass spectrometer and detected as a mass spectrum. ${ }^{27}$

SIMS analysis may be performed with two different mass analyzers: time-of-flight $(\mathrm{ToF})^{28}$ and magnetic sector. ${ }^{29}$ The ToF-SIMS technique allows recording of a mass spectrum at each pixel for known and unknown ion species and thus represents a label-free imaging technique. However, its lateral resolution of $\sim 200 \mathrm{~nm}$ and lower secondary ion yields during high-spatial resolution acquisition are limiting factors for the application for subcellular imaging.

\subsection{NanoSIMS Technique for High-Resolution Imaging}

Nowadays, a promising technique for high-resolution chemical imaging has been magnetic sector SIMS, which provides higher

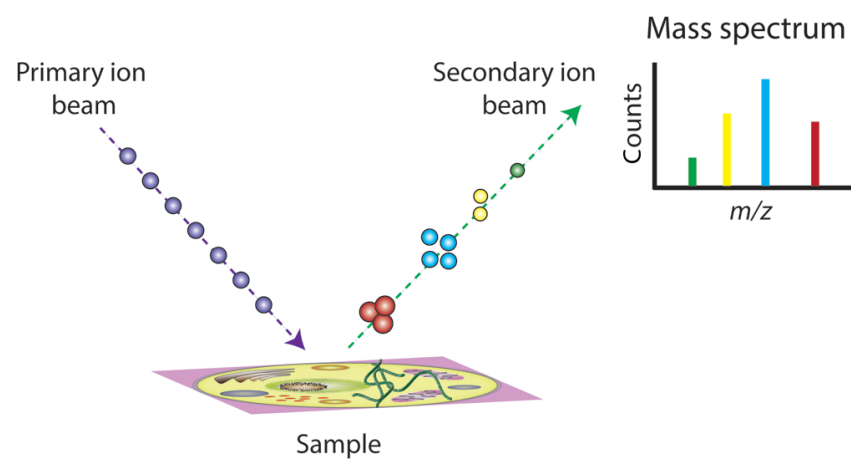

Fig. 3 Schematic representation of the sputtering process in SIMS. Primary ion beam (monoatomic or cluster ion species of metals, gases, or carbon) is scanned over the sample surface and sputters away secondary species (neutrals, electrons, and ions). Only charged species $(\sim 1 \%)$ will be extracted to the mass spectrometer and analyzed. ${ }^{25}$ secondary ion yields in comparison to ToF-SIMS. Commercial versions of magnetic sector SIMS in existence today are Cameca NanoSIMS 50 or 50L instruments (Fig. 4).

NanoSIMS is a dynamic SIMS instrument with the ability to analyze most of the elements in the periodic table. Continuous primary ion bombardment allows breakage of molecular bonds in the sample while continuously generating usually monoatomic and diatomic secondary ions, therefore, molecules of interest must have a specific element or have to be isotopically labeled. The unique feature of this instrument is the presence of coaxial ion optics, which enables copropagation of primary and secondary ions. This design allows ion optics to be close and perpendicular to the sample, thus reducing the primary ion beam spot size and increasing the lateral resolution and the secondary ion collection efficiency (transmission). Due to the

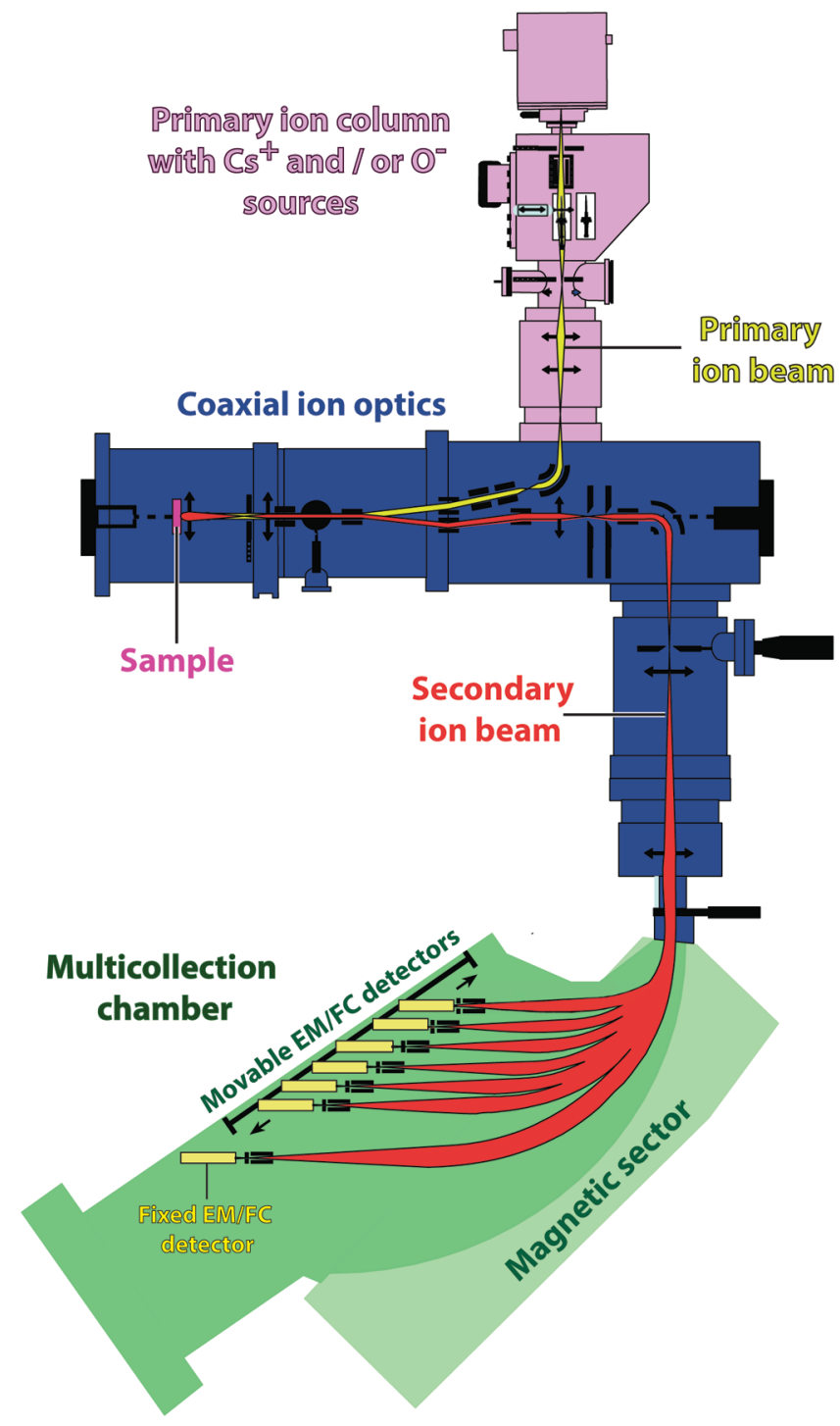

Fig. 4 Scheme of NanoSIMS 50L showing the main parts of the instrument. The primary ion beam consisting of $\mathrm{Cs}^{+}$or $\mathrm{O}^{-}$species is focused onto the sample. Coaxial ion optics allows reduction of the primary ion beam spot size and therefore improving the lateral resolution. Secondary ion species are separated in the dual-focusing sector-field mass analyzer and collected in parallel with five (NanoSIMS 50) or seven detectors (NanoSIMS 50L) in the multicollection chamber. EM, electron multiplier and FC, Faraday cup. Original schematics by courtesy of CAMECA. 
coaxial design of optics, the primary and secondary ion beams should be opposite polarities. Primary ion sources commercially available are either cesium sources, which generate negative secondary ions and can be focused down to 30 - to 50 -nm beam diameter, or duoplasmatron sources for $\mathrm{O}^{-}$primary ion probes with 40-nm diameter (newest model of the source), which generates positive secondary ions. The secondary ions are separated according to their mass to charge ratio $(\mathrm{m} / \mathrm{z})$ in the dual-focusing sector mass analyzer first with an electrostatic filter and further with a magnetic sector. Double focusing capability of the mass spectrometer allows the NanoSIMS instrument imaging with high mass resolution. Unlike ToF-SIMS, NanoSIMS can detect only up to seven species simultaneously at electron multiplier or Faraday cup detectors. ${ }^{27,30}$

The NanoSIMS imaging technique has proved to be useful in a wide range of research fields, such as material science, ${ }^{31}$ biological geochemistry and cosmochemistry, ${ }^{30}$ plant research, ${ }^{32}$ environmental microbiology, ${ }^{33}$ cell biology, ${ }^{34}$ and others. An outstanding spatial resolution has allowed it to be applied in biological studies, specifically, imaging cells and their compartments. ${ }^{35}$ In such studies, a $\mathrm{Cs}^{+}$primary ion beam of $16-\mathrm{keV}$ energy is usually applied, and stable isotopes, such as ${ }^{2} \mathrm{H},{ }^{13} \mathrm{C}$, ${ }^{15} \mathrm{~N}$, and ${ }^{18} \mathrm{O}$ are used for labeling of target molecules. Owing to excellent mass resolution with high transmission, NanoSIMS is capable of resolving ion species, such as ${ }^{13} \mathrm{C}^{12} \mathrm{C}^{-}$and ${ }^{12} \mathrm{C}_{2}^{-}$, and even isobars with the same nominal mass, such as ${ }^{12} \mathrm{C}^{15} \mathrm{~N}^{-}$and ${ }^{12} \mathrm{C}^{14} \mathrm{~N}^{1} \mathrm{H}$. It operates in an ultrahigh vacuum environment and thus necessitates adequate preparation of water-rich samples. Therefore, the preparation of biological specimens requires attention due to the possible migration or complete loss of soluble compounds during fixation and embedding phases. ${ }^{30}$

NanoSIMS has proven to be capable of imaging proteins by pulsing isotopically labeled amino acids ${ }^{36}$ or nucleotides ${ }^{35}$ into the cell and imaging cellular turnover. However, understanding metabolism at the subcellular level, gene, and protein expression, the study of protein complexes and single organelles are still limited as this technique cannot provide chemical maps of specific proteins. ${ }^{34,36}$ Attempts to improve the specificity of the protein imaging have been done by Angelo et al., ${ }^{37}$ where NanoSIMS was used for imaging cells labeled with metal conjugated antibodies. Primary antibodies were coupled with up to 100 isotopically pure lanthanides, and positive secondary ions of metals were sputtered away with an oxygen primary ion beam. The chemical distribution of different lanthanide elements corresponded to a specific antibody and therefore to a specific epitope. Although this methodology allows single-stepspecific protein analysis without the need to correlate with other imaging technique(s), it still suffers from several pitfalls, such as difficulty in targeting some epitopes with antibodies, ${ }^{37}$ and reduction of accurate imaging as epitopes should be labeled at high density and metal-labeled antibodies react with only a small portion of epitopes and thus incorporate rather poorly into the biological sample. ${ }^{38}$

As for super-resolution optics, NanoSIMS is limited in terms of both contrast and resolution. The former depends on the number of ions that can be determined from the particular structure. For example, a gold particle of $\sim 50$ to $100 \mathrm{~nm}$ in diameter will provide excellent contrast, ${ }^{36}$ but small particles, such as the 5nm ones used commonly in electron microscopy, are extremely difficult to detect. At the same time, the image contrast depends on the difference in the abundances of the isotopes measured between the structure of interest and the surrounding material.
For example, ${ }^{13} \mathrm{C}$ has been used as a common isotope for labeling proteins in biological samples. ${ }^{13} \mathrm{C}$ is present at an abundance of $\sim 1.05 \%$ of all $\mathrm{C}$ atoms in all biological structures and thus provides a strong background signal, which reduces the contrast. ${ }^{15} \mathrm{~N}$ should be used whenever possible for protein labeling, since it is normally far less abundant $(\sim 0.36 \%)$ and thus provides better contrast.

The image resolution in the lateral $(X-Y)$ plane depends mainly on the primary beam thickness. A beam of $\sim 50 \mathrm{~nm}$ results in a comparable resolution ( 35 to $50 \mathrm{~nm}$ ), since it produces isotopes only from this small area. The axial resolution has been debated, albeit it seems to be identical to the amount of material ablated by the primary beam during scanning. In biological samples, this has been determined to be in the order of 10 to $20 \mathrm{~nm} .^{36}$ The main limitation in this type of experiment is the sample drift, which should be minimized as it otherwise distorts both the lateral and the axial measurements.

\section{Correlated Imaging Techniques}

The obstacles in identifying specific subcellular features, such as organelles, and the difficulty in obtaining the specificity of protein imaging generated the idea of combining different imaging techniques. Several research groups have made an effort to combine electron and super-resolution microscopy to localize fluorescently tagged proteins to subcellular compartments. For example, Jorgensen and collaborators developed a methodology to correlate confocal, STED, and PALM microscopy with secondary electron micrographs. They performed super-resolution microscopy on ultrathin sections of fixed Caenorhabditis elegans to localize fluorescently tagged proteins and further perform electron microscopy on the same sections. This approach allowed correlation between protein localization and ultrastructural features of the sample revealed in electron micrographs (Fig. 5). ${ }^{39}$

To reveal the subcellular localization of target molecules with chemical information, Takado et al. ${ }^{40}$ performed a set of experiments to correlate NanoSIMS imaging data and transmission electron microscopy (TEM) micrographs, both obtained from the same specimen. Their work was related to the investigation of glycogen metabolism in liver and brain tissues by administrating the uniformly ${ }^{13} \mathrm{C}$-labeled glucose into mice. After suitable sample preparation, TEM imaging was performed and consequently NanoSIMS imaging of the same section area (Fig. 6). This allowed precise subcellular localization of glucosyl residues and their quantitation as a ${ }^{13} \mathrm{C} /{ }^{12} \mathrm{C}$ isotopic ratio at nanometer scale.

To take a step forward in identifying specific organelles and proteins and at the same time to obtain correlative chemical information, Rizzoli et al. ${ }^{36}$ developed a methodology where STED and NanoSIMS techniques were combined, and this imaging approach has been named correlated optical isotopic nanoscopy (COIN). Due to the difficulties of NanoSIMS in identifying specific subcellular structures and proteins, the necessity to use super-resolution microscopy, such as STED, had emerged. This nanoscopic approach has allowed the investigation of the turnover of different cellular structures, such as membranes, organelles, and protein clusters.

This type of experiment proceeds as follows. To identify the turnover of specific classes of molecules, such as proteins, lipids, or nucleic acids, the cells or organisms are fed with specific metabolites labeled with rare isotopes, such as ${ }^{13} \mathrm{C}$ or ${ }^{15} \mathrm{~N}$. Some of the most popular metabolites are the amino acids ${ }^{13} \mathrm{C}$-lysine 

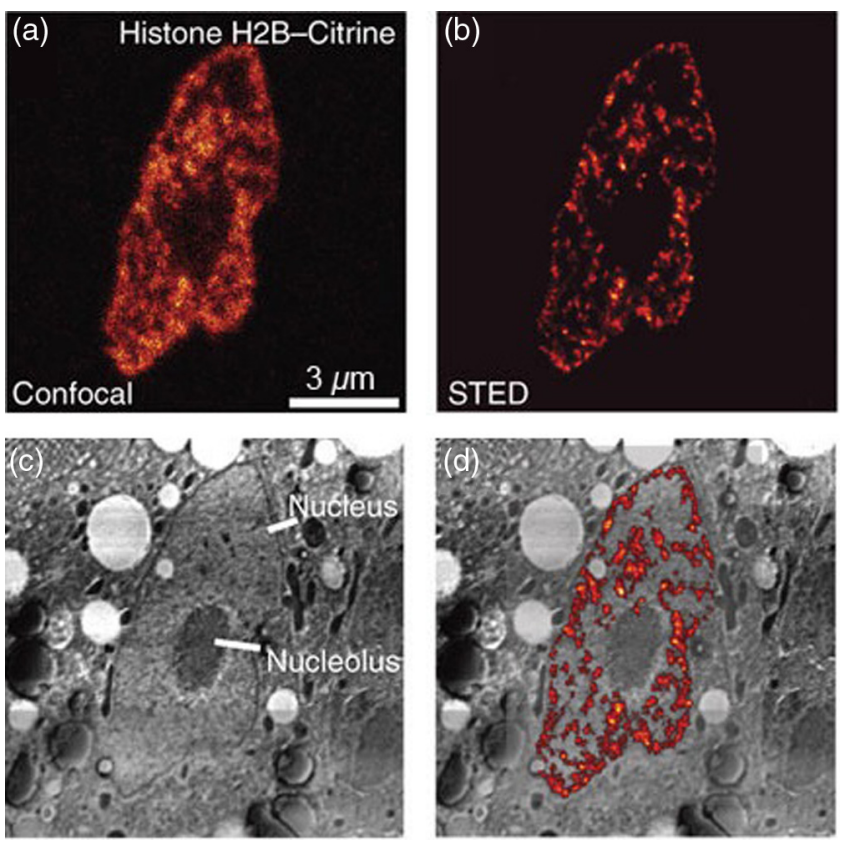

Fig. 5 Histone proteins in ultrathin section of $C$. elegans imaged with confocal, STED, and SE microscopy. (a) Confocal image, (b) STED image, (c) SE micrograph, and (d) an overlay of STED [in (b)] and EM image [in (c)] showing the precise localization of histones. ${ }^{39}$ Figure was reprinted with permission from Ref. 39. (c) 2010, Macmillan Publishers Ltd.

and ${ }^{15} \mathrm{~N}$-leucine, which are incorporated into proteins, and therefore label all of the newly produced proteins. Protein turnover in specific areas can thus be imaged by scanning them in NanoSIMS and measuring the ratio of newly produced proteins (labeled by ${ }^{13} \mathrm{C}$ or ${ }^{15} \mathrm{~N}$ ) to older proteins (labeled by the common isotopes, $\left.{ }^{12} \mathrm{C},{ }^{14} \mathrm{~N}\right)$. Other popular labels include ${ }^{15} \mathrm{~N}$-thymidine, which is a building block of nucleic acids, or ${ }^{18} \mathrm{O}$-cholesterol, which enables the investigation of the turnover of cholesterolcontaining lipid membranes.

One such experiment is shown in Fig. 7, focusing on cells from blood vessels of mice fed with ${ }^{13} \mathrm{C}$-lysine for two weeks. Five blood cells can be detected in three capillaries, two of which exhibit no new proteins (no ${ }^{13} \mathrm{C}$ labeling) and three of which exhibit strong labeling. The explanation is a simple one: the five cells are, judging by their morphology, erythrocytes, which lack nuclei, and therefore have no protein turnover. They obtain their entire protein contents during their formation and then keep it until their degradation. The cells lacking ${ }^{13} \mathrm{C}$ labeling had been produced before feeding the animals with this isotope, therefore they cannot contain any ${ }^{13} \mathrm{C}$-labeled proteins. In contrast, the three strongly labeled cells had been produced at a stage when the animal's diet contained ${ }^{13} \mathrm{C}$, and they thus contain exclusively metabolically new proteins.

One problem with this experiment is that the nature of the investigated structures can only be guessed from their morphology, which is difficult especially for subcellular organelles. To address this issue, specific proteins are immunostained and are identified in confocal or STED microscopy, and then the samples are imaged in NanoSIMS, which is the basis of the COIN procedure mentioned above. This procedure enables the investigators to analyze by NanoSIMS the turnover within specific areas identified in fluorescence imaging, as demonstrated in Fig. 8.

Even though the COIN approach makes it possible to study the cellular turnover in a variety of biological systems, there are some restrictions of the methodology, such as the necessity to use two rather expensive instruments, the difficulty of antibodies to incorporate into the specimen, and finding a sufficient number of epitopes to obtain accurate nanoscopic imaging. Recently, Vreja et al. ${ }^{41}$ showed that bypassing the immunocytochemistry is possible via in-situ hybridization. In this study, an elegant approach for specific isotopic and fluorescent protein labeling has been developed with genetic-code expansion and afterward chemoselective click-chemistry labeling (Fig. 9).

This labeling approach comprises the application of an unnatural amino acid (UAA), such as propargyl-1-lysine (PRK), which can be incorporated into the protein of interest. Its DNA had been previously modified to harbor an Amber STOP codon (TAG), which determines the binding site for the UAA and pair of tRNA and aminoacyl-tRNA synthetase with a (a)

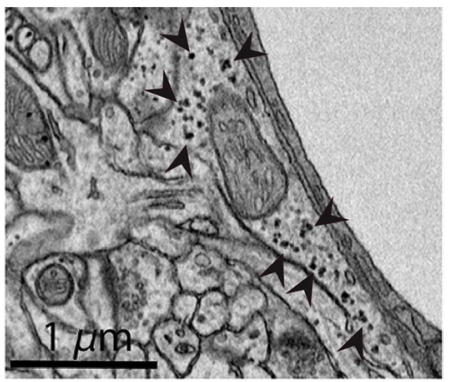

(b)

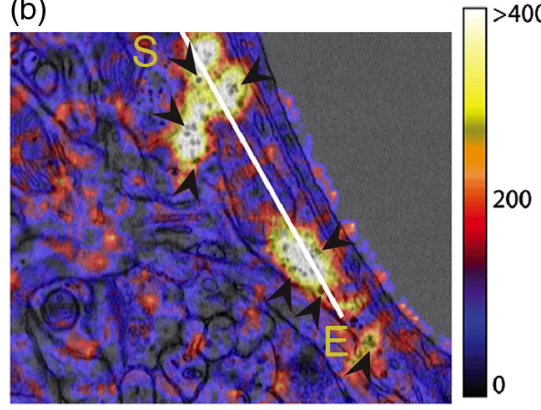

$\delta^{13} \mathrm{C}(\% \mathrm{o})$

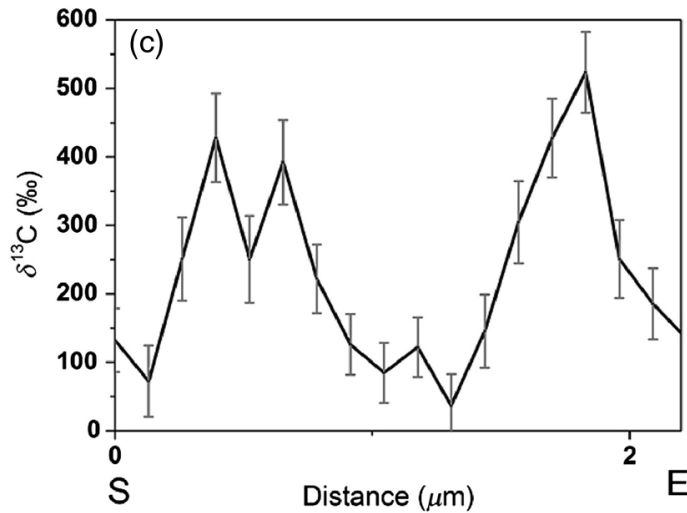

Fig. 6 The synthesis of mouse brain glycogen from uniformly labeled $\left[\mathrm{U}-{ }^{13} \mathrm{C}\right]$ glucose investigated by correlated electron microscopy and SIMS imaging. (a) TEM image of brain section (arrows in black indicate glycogen granules), (b) chemical map showing ${ }^{13} \mathrm{C}$ enrichment of the same area as in the TEM image in (a). The ${ }^{13} \mathrm{C}$ enrichment is expressed as the increase (in \%o) over the natural abundance of ${ }^{13} \mathrm{C}$ in tissues. The incorporation of $\left[\mathrm{U}-{ }^{13} \mathrm{C}\right]$ contributes to a far higher $\% \circ$ of ${ }^{13} \mathrm{C}$ in the regions containing the glycogen. (c) Line profile of ${ }^{13} \mathrm{C}$ enrichment. A line was drawn over several glycogen-containing regions, as indicated in image (b), ${ }^{40}$ and the $\% \circ$ of ${ }^{13} \mathrm{C}$ was measured, as indicated in this panel. $S$ and $E$ indicate the start and end of the line scan. Reprinted with permission from Ref. 40. (c) 2015, Elsevier. 

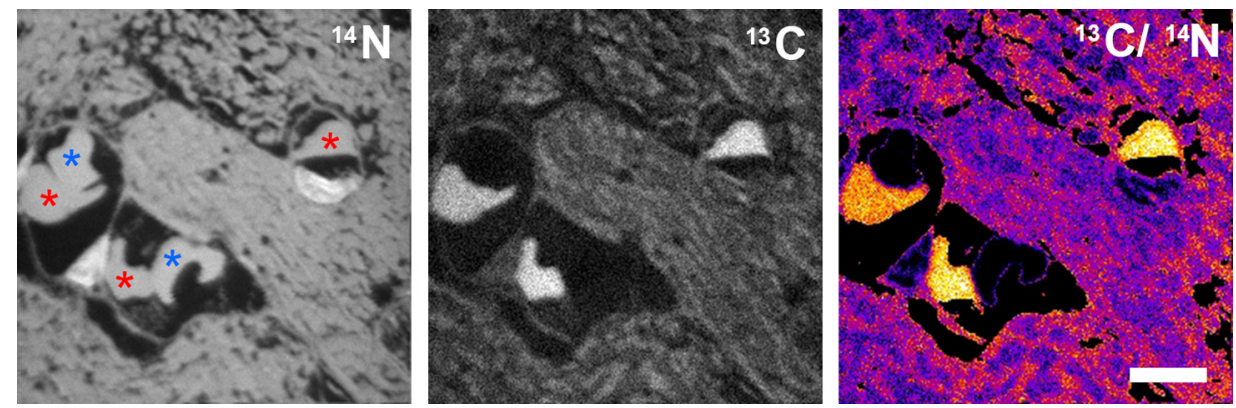

Fig. 7 Protein turnover in the mouse heart measured by SIMS. Mice were fed for two weeks with ${ }^{13} \mathrm{C}$-labeled leucine and an area of the heart that contains three blood vessels (capillaries) was imaged. The images show ${ }^{14} \mathrm{~N}$ (all proteins), along with ${ }^{13} \mathrm{C}$ (newly synthesized proteins), and a ratio image. The red stars point to three blood cells, found within the capillaries, which show a very strong ${ }^{13} \mathrm{C}$ labeling -in fact, stronger than all of the rest of the tissue. They share the space in the capillaries with two other blood cells that have no discernible ${ }^{13} \mathrm{C}$ labeling (blue stars). These points to the presence of cells with widely different metabolisms, or widely different ages, in the blood stream. Scale bar: $4 \mu \mathrm{m}$.

(a)

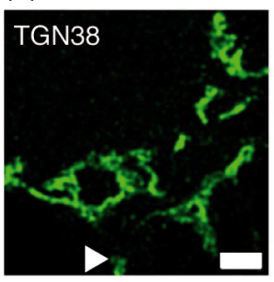

(b)

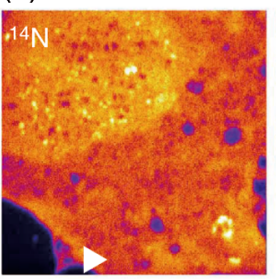

$04998147 \quad 196$
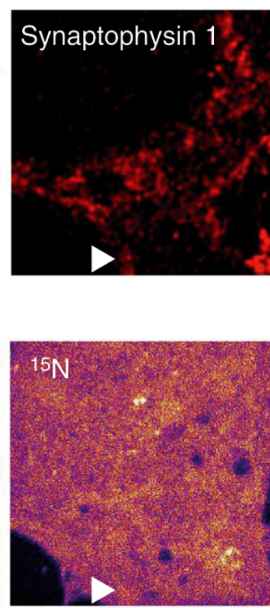

$\begin{array}{lllll}0 & 5 & 11 & 16 & 21\end{array}$
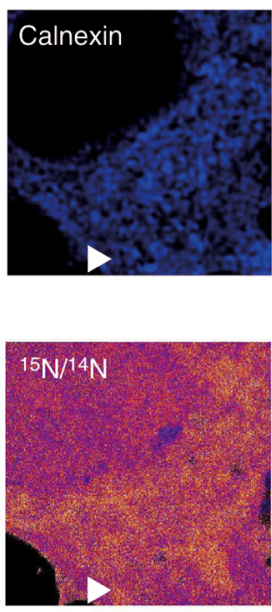

$\begin{array}{llll}0 & 0.06 & 0.13 & 0.19\end{array}$
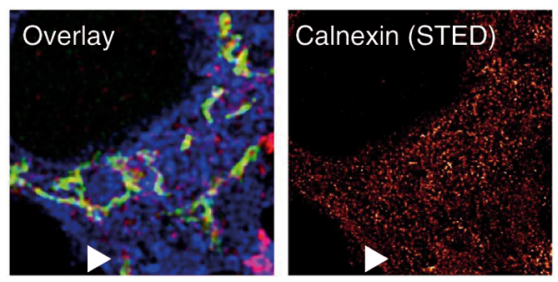

(c)

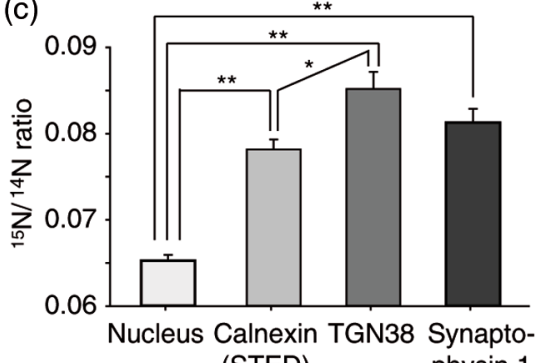

Fig. 8 COIN of immunocytochemically and isotopically labeled proteins in a neuronal cell body. Neurons in culture were fed with ${ }^{15} \mathrm{~N}$-leucine, to measure protein turnover, and were then fixed and immunostained for different organelle markers. From left to right: (a) confocal images of different organelle markers: TNG38 for the golgi apparatus, synaptophysin 1 for precursor vesicles, and calnexin for the endoplasmic reticulum. The rightmost panel shows an STED image for the calnexin label. (b) NanoSIMS chemical maps of the same area: ion images ${ }^{12} \mathrm{C}^{14} \mathrm{~N}-,{ }^{12} \mathrm{C}^{15} \mathrm{~N}$-, and ${ }^{15} \mathrm{~N}$-enrichment, respectively. (c) The bar plot showing ${ }^{15} \mathrm{~N}$-enrichment in the different regions of the neuron, identified by the different markers. ${ }^{36}$

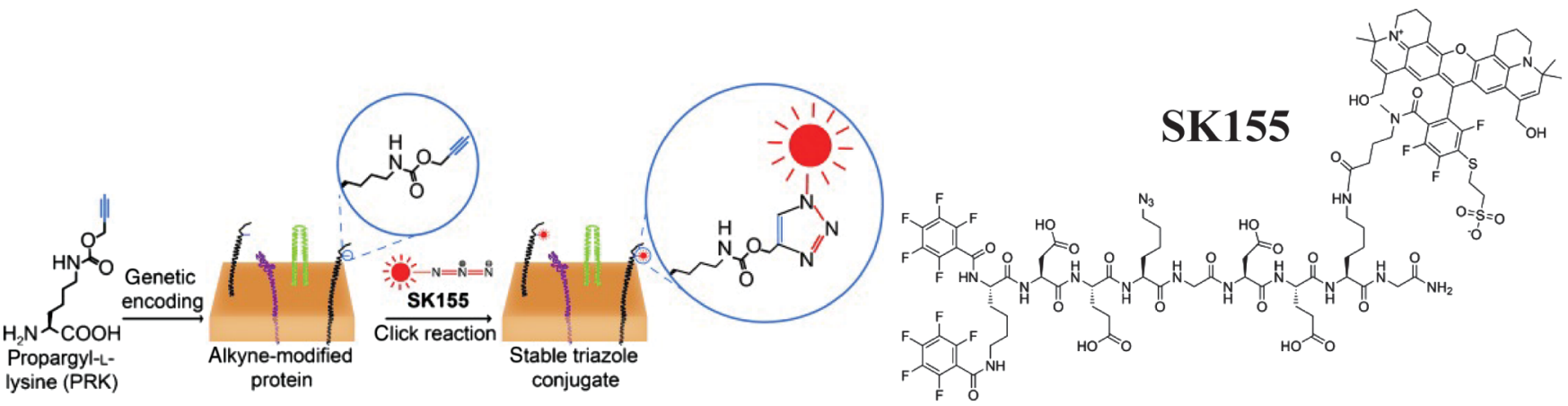

Fig. 9 Schematic representation of genetic-code expansion via UAAs. PRK was introduced into the protein of interest and labeled via click reaction with the probe SK155, which can be used in both fluorescence and NanoSIMS imaging. ${ }^{41}$ 
(a)

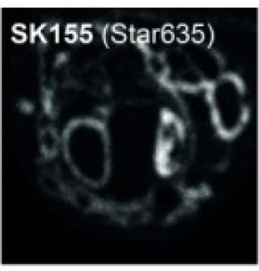

(b)
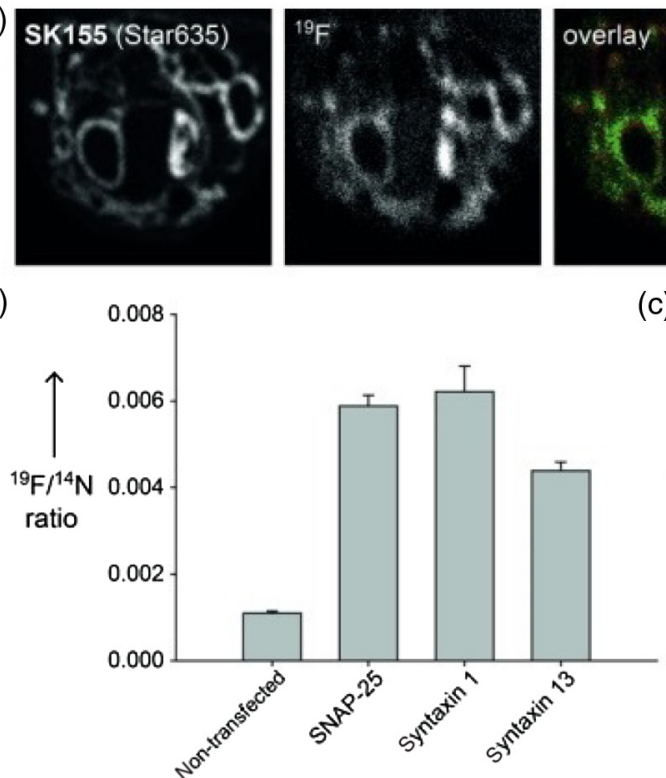

(c)
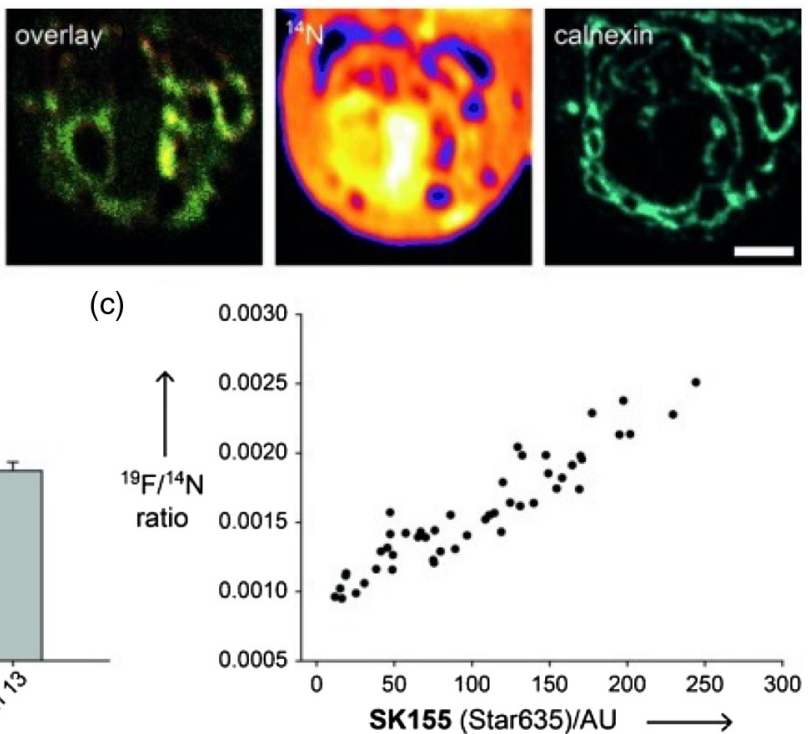

Fig. 10 Imaging data for several genetically encoded proteins in baby hamster kidney fibroblasts. (a) Images of the tagged protein syntaxin, from left to right: confocal image of Star635; NanoSIMS ion image of ${ }^{19} \mathrm{~F}$ - distribution; overlay of Star635 signal (red) and ${ }^{19} \mathrm{~F}$ - (green); NanoSIMS ion image of ${ }^{12} \mathrm{C}^{14} \mathrm{~N}$ - ion species revealing cellular features; confocal image of immunostained protein calnexin. (b) The isotopic ratio $\left({ }^{19} \mathrm{~F}-/{ }^{14} \mathrm{~N}-\right)$ of nontransfected and transfected cells was significantly higher for all expressed proteins: SNAP-25 $(p<0.01)$, syntaxin $1(p<0.001)$, and syntaxin $13(p<0.001 ; t$-tests). (c) The signal intensity for the isotopic ratio $\left({ }^{19} \mathrm{~F}-1{ }^{14} \mathrm{~N}-\right)$ increases proportionally to the Star635 signal in the corresponding cell regions, suggesting that labeling procedure was efficient. ${ }^{41}$

role to introduce the UAA into the specific protein. The UAA is revealed after cellular fixation when a specific probe is introduced containing both fluorophore and isotope atoms. In this work, such a probe was SK155, dipentafluorobenzyl peptide, containing ${ }^{19} \mathrm{~F}$ atoms for NanoSIMS imaging, the fluorophore Abberior Star635 for testing the probe with fluorescence microscopy and the azide group, which allowed click chemistry with
PRK (Fig. 9). Figure 10 shows the imaging data for proteins from mammalian cells modified with PRK and labeled with SK155.

Although the choice of fluorine as an isotopic label has proven to be suitable for NanoSIMS imaging due to its low abundance in biological systems, it has been shown to interfere in fluorescence acquisition. Thus, recently, a

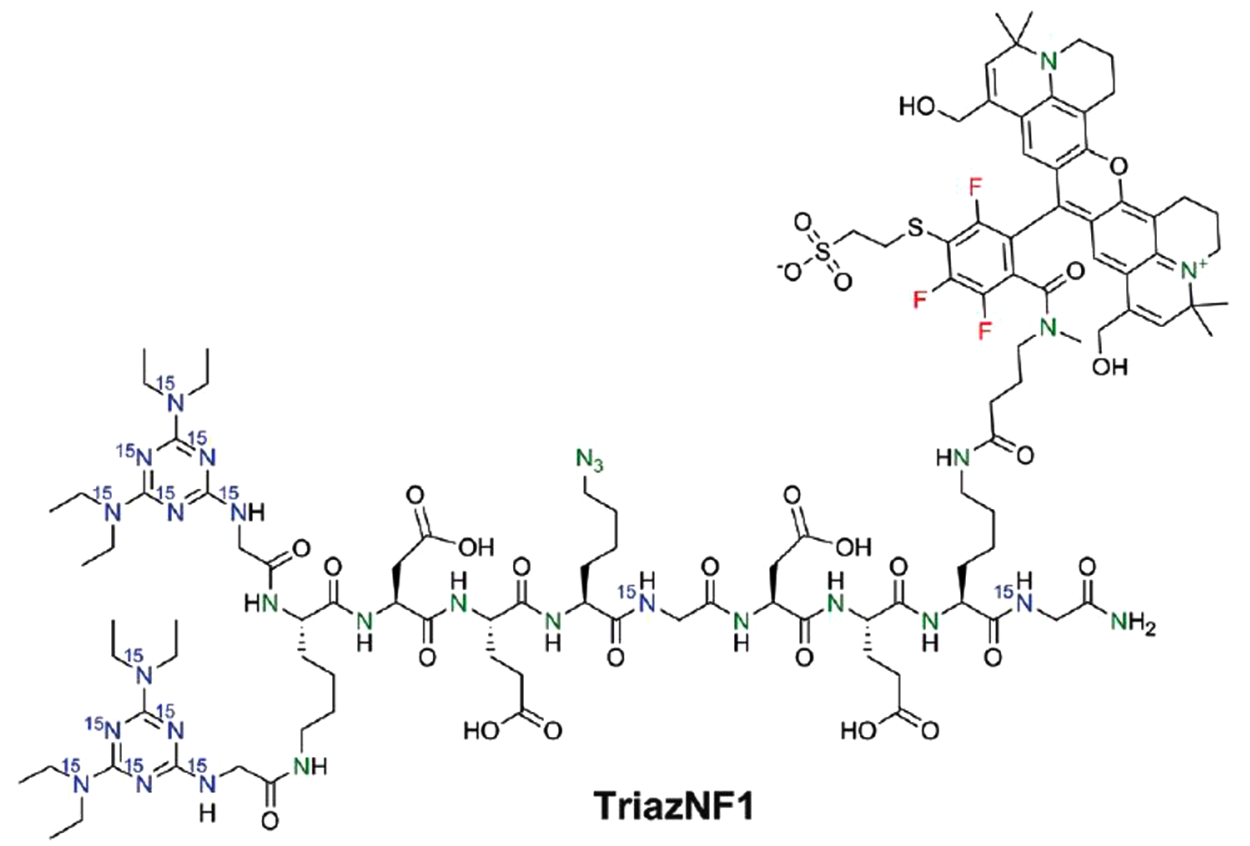

Fig. 11 TriazNF1 dual probe has an azide group for click chemistry and the fluorescent probe, Star635, as SK155 but additionally ${ }^{15} \mathrm{~N}$ atoms (marked in blue) are incorporated for NanoSIMS imaging. ${ }^{42}$ 
probe TriazNF1 (Fig. 11) similar to SK155 has been synthesized having a high ${ }^{15} \mathrm{~N} /{ }^{14} \mathrm{~N}$ ratio for NanoSIMS imaging and at the same time allowing easier fluorescence imaging if that is a demand. ${ }^{42}$

There is a wide spectrum of approaches to imaging biological systems, but only a few imaging techniques in a correlation allow the identification of subcellular features and reveal the spatial distribution of organelles and their components. COIN is one way to obtain such high-resolution imaging data. The usefulness of the COIN approach is that combined techniques provide complementary information: SIMS strives to understand the chemical composition, while STED microscopy reveals the structural information, thus COIN allows a highly precise description of turnover mechanisms for specific biomolecules. Further, organic chemistry has opened a door to genetically encoded target labeling, which is a sophisticated approach for specific protein labeling. This methodology will probably find a wide range of application, as geneticcode expansion is possible to achieve in both prokaryotic and eukaryotic cells.

\section{Conclusions}

We conclude that both super-resolution optical microscopy and SIMS have matured over the last few years and have reached a level that renders feasible the wide application to the investigation of biological questions. Several problems still persist, including the difficulty of fitting in one setup the different requirements of mass spectrometry or optical imaging. For example, although live imaging was the initial goal of all optical techniques, this is still not feasible for combined optical and isotopic nanoscopy, since the high vacuum used in NanoSIMS precludes the use of living specimens. The reliance on fixed samples is not optimal, since fixation artifacts may disrupt the cellular elements under investigation. A solution to this problem is the use of live optical imaging, followed by high-pressure freezing and freeze-substitution, which enables a combination of analyzing the dynamics of the living sample, followed by an investigation of its structure by any technique that requires vacuum, including SIMS and electron microscopy. Ultimately, a cryostage will be invaluable for COIN imaging so that the living specimen can be immediately frozen and investigated by SIMS, without any disruption caused by plastic embedding.

\section{Disclosures}

The authors declare no competing financial interests.

\section{Acknowledgments}

All figures are used by permission. Figure 8 is reused from Ref. 36, which is licensed under Creative Commons Attribution-Noncommercial-ShareAlike 3.0 Unported License. https:// creativecommons.org/licenses/by-nc-sa/3.0/legalcode. Figures 9 and 10 are reprinted from Ref. 41, which are licensed under Creative Commons Attribution Noncommercial NoDerivs License. https://creativecommons.org/licenses/by-nc-nd/3.0/ legalcode. Figure 11 is reused from Ref. 42 and reproduced by permission of The Royal Society of Chemistry. This work was funded by a Consolidator Grant from the European Research Council (NeuroMolAnatomy, to S. O. Rizzoli).

\section{References}

1. A. Dittrich, E. Siewert, and F. Schaper, "Determination of protein turnover rates in the JAK/STAT pathway using a radioactive pulse-chase approach," Methods Mol. Biol. 967, 69-80 (2013).

2. K. I. Willig et al., "STED microscopy reveals that synaptotagmin remains clustered after synaptic vesicle exocytosis," Nature 440(7086), 935-939 (2006).

3. G. Donnert et al., "Two-color far-field fluorescence nanoscopy," Biophys. J. 92(8), L67-L69 (2007).

4. S. W. Hell and J. Wichmann, "Breaking the diffraction resolution limit by stimulated emission: stimulated-emission-depletion fluorescence microscopy," Opt. Lett. 19(11), 780-782 (1994).

5. S. W. Hell, M. Dyba, and S. Jakobs, "Concepts for nanoscale resolution in fluorescence microscopy," Curr. Opin. Neurobiol. 14, 599-609 (2004).

6. T. A. Klar et al., "Fluorescence microscopy with diffraction resolution barrier broken by stimulated emission," PNAS 97(15), 8206-8210 (2000).

7. V. Westphal and S. W. Hell, "Nanoscale resolution in the focal plane of an optical microscope," Phys. Rev. Lett. 94(14), 143903 (2005).

8. G. Donnert et al., "Macromolecular-scale resolution in biological fluorescence microscopy," PNAS 103(31), 11440-11445 (2006).

9. J. Tønnesen et al., "Two-color STED microscopy of living synapses using a single laser-beam pair,” Biophys. J. 101(10), 2545-2552 (2011).

10. U. V. Nägerl et al., "Live-cell imaging of dendritic spines by STED microscopy," PNAS 105(48), 18982-18987 (2008).

11. P.A. Pellett et al., "Two-color STED microscopy in living cells," Biomed. Opt. Express 2(8), 2364-2371 (2011).

12. V. Westphal et al., "Video-rate far-field optical nanoscopy dissects synaptic vesicle movement," Science 320(5873), 246-249 (2008).

13. E. Sezgin et al., "A comparative study on fluorescent cholesterol analogs as versatile cellular reporters," Lipid Res. 57(2), 299-309 (2016).

14. M. J. Rust, M. Bates, and X. Zhuang, "Stochastic optical reconstruction microscopy (STORM) provides sub-diffraction-limit image resolution," Nat. Methods 3(10), 793-796 (2006).

15. S. A. Jones et al., "Fast three-dimensional super-resolution imaging of live cells," Nat. Methods 8(6), 499-508 (2011).

16. E. Betzig et al., "Imaging intracellular fluorescent proteins at nanometer resolution," Science 313(5793), 1642-1645 (2006).

17. B. Dani et al., "Super-resolution imaging of chemical synapses in the brain," Neuron 68(5), 843-856 (2010).

18. A. Huang et al., "Three-dimensional super-resolution imaging by stochastic optical reconstruction microscopy," Science 319(5864), 810-813 (2008).

19. H. Shroff et al., "Live-cell photoactivated localization microscopy of nanoscale adhesion dynamics," Nat. Methods 5(5), 417-423 (2008).

20. F. Huang et al., "Video-rate nanoscopy using sCMOS camera-specific single-molecule localization algorithms," Nat. Methods 10(7), 653-658 (2013).

21. H. Ta et al., "Mapping molecules in scanning far-field fluorescence nanoscopy," Nat. Commun. 13(6), 7977 (2015).

22. K. C. Schmidt and C. B. Smith, "Resolution, sensitivity and precision with autoradiography and small animal positron emission tomography: implications for functional brain imaging in animal research," Nucl. Med. Biol. 32, 719-725 (2005).

23. M. Karas, D. Bachmann, and F. Hillenkamp, "Influence of the wavelength in high-irradiance ultraviolet laser desorption mass spectrometry of organic molecules," Anal. Chem. 57, 2935-2939 (1985).

24. M. M. Gessel, J. L. Norris, and R. M. Caprioli, "MALDI imaging mass spectrometry: spatial molecular analysis to enable a new age of discovery," J. Proteomics 107, 71-82 (2014).

25. K. Chughtai and R. M. A. Heeren, "Mass spectrometric imaging for biomedical tissue analysis," Chem. Rev. 110, 3237-3277 (2010).

26. E. G. Solon et al., "Autoradiography, MALDI-MS, and SIMS-MS imaging in pharmaceutical discovery and development," AAPS J. 12, 11-26 (2009).

27. S. G. Boxer, M. L. Kraft, and P. K. Weber, "Advances in imaging secondary ion mass spectrometry for biological samples," Ann. Rev. Biophys. 38, 53-74 (2009).

28. A. Benninghoven, "Chemical analysis of inorganic and organic surfaces and thin films by static time-of-flight secondary ion mass spectrometry (TOF-SIMS)," Angew. Chem. Int. Ed. Eng. 33, 1023-1043 (1994). 
29. G. S. R. Castaing, "Microanalyse par émission ionique secondaire," J. Microsc. 1, 395-410 (1962).

30. P. Hoppe, S. Cohen, and A. Meibom, "NanoSIMS: technical aspects and applications in cosmochemistry and biological geochemistry," Geostand. Geoanal. Res. 37, 111-154 (2013).

31. S. Lozano-Perez et al., "High-resolution imaging of complex crack chemistry in reactor steels by NanoSIMS," J. Nucl. Mater. 374, 61-68 (2008).

32. K. L. Moore et al., "Elemental imaging at the nanoscale: NanoSIMS and complementary techniques for element localisation in plants," Anal. Bioanal. Chem. 402, 3263-3273 (2011).

33. F. Inagaki et al., "Exploring deep microbial life in coal-bearing sediment down to 2.5 km below the ocean floor," Science 349, 420-424 (2015).

34. M. L. Steinhauser et al., "Multi-isotope imaging mass spectrometry quantifies stem cell division and metabolism," Nature 481, 516-519 (2012).

35. H. Brismar et al., "Study of protein and RNA in dendritic spines using multi-isotope imaging mass spectrometry (MIMS)," Surf. Interface Anal. 46, 158-160 (2014).
36. S. K. Saka et al., "Correlated optical and isotopic nanoscopy," Nat. Commun. 5, 8 (2014).

37. M. Angelo et al., "Multiplexed ion beam imaging of human breast tumors," Nat. Med. 20, 436-442 (2014).

38. F. Opazo et al., "Aptamers as potential tools for super-resolution microscopy," Nat. Methods 9, 938-939 (2012).

39. S. Watanabe et al., "Protein localization in electron micrographs using fluorescence nanoscopy," Nat. Methods 8, 80-84 (2011).

40. Y. Takado et al., "Imaging liver and brain glycogen metabolism at the nanometer scale," Nanomed. Nanotechnol. Biol. Med. 11, 239-245 (2015).

41. I. C. Vreja et al., "Secondary-ion mass spectrometry of genetically encoded targets," Angew. Chem. Int. Ed. 54, 5784-5788 (2015).

42. S. Kabatas et al., "A contamination-insensitive probe for imaging specific biomolecules by secondary ion mass spectrometry," Chem. Commun. 51, 13221-13224 (2015).

Biographies for the authors are not available. 